\title{
The Mobile Spatial coordinate Measuring System II (MScMS-II): system description and preliminary assessment of the measurement uncertainty
}

\author{
M. Galetto ${ }^{1 \star}$, L. Mastrogiacomo, and B. Pralio \\ Politecnico di Torino, Production Systems and Business Economics Department (DISPEA), Corso Duca degli Abruzzi, \\ 24 - I-10129 Torino, Italy
}

Received: 5 August 2010 / Accepted: 20 August 2010

\begin{abstract}
According to the increasing interest in metrological systems for the dimensional measurements of large-size objects in a wide range of industrial sectors, several solutions based on different technologies, working principles, architectures, and functionalities have recently been developed. Among all, the most flexible and easily transportable solutions are those that have aroused most interest and have found greater success. In order to address the needs of Large-Scale Metrology (LSM) applications, a distributed flexible system based on a network of low-cost InfraRed (IR) sensors - the Mobile Spatial coordinate Measuring System II (MScMS-II) - has been developed at the Industrial Quality and Metrology Laboratory of Politecnico di Torino. This paper presents a preliminary uncertainty assessment of the system referring to the measured point coordinates in the 3D space, focusing on the sources of measurement uncertainty and the related propagation laws. A preliminary metrological characterization of MScMS-II architecture, experimentally evaluated through a system prototype, is also presented and discussed.
\end{abstract}

Keywords: MScMS-II; dimensional measurement; uncertainty assessment; metrological characterization; photogrammetry; Large-Scale Metrology

\section{Introduction}

The ever increasing interest in metrology for large-sized objects, since its definition as "Large-Scale Metrology" (LSM) given by Puttock in 1978 [1], has been responsible for the wide variety of measurement systems. Different technologies, working principles, functionalities and architectures have been implemented, in order to fulfil the requirements of the several industrial sectors involved [2,3].

At present, optical-based systems clearly demonstrate their advantages over the other approaches and their potentialities for LSM applications [2]. As noted by Estler et al. [4] in a recent state-of-the-art update, tremendous improvements have been achieved in this field due to advancements in optical technology and fast, low-cost computation. However, it is recognized that significant technical challenges still remain "associated with high accuracy measurements of large structures" [4]. Furthermore, most of these systems may not be cost-effective for measurements below a given level of accuracy [5].

Developments in imaging technology, which afforded the community large area CCD sensors, and improvements of target image location algorithms have led to an ever-increasing competitiveness of vision-based metrology. As a matter of fact, different well-settled solutions based

^ Correspondence: maurizio.galetto@polito.it on photogrammetry are commercially available, providing accurate, portable, and versatile instruments for threedimensional coordinate measurement $[6,7]$. The fundamental principle used by photogrammetry is triangulation. By taking photographs or video images from at least two different positions, it is possible to reconstruct the spatial location of a point and, therefore, the geometry or the main features of an object. Notwithstanding their multiple sensor-based structure, the existing photogrammetric instruments are applied to reduced working volumes and do not exploit the potentialities of a wireless sensor network layout.

This paper presents a novel IR-based distributed system (MScMS-II - Mobile Spatial coordinate Measuring System II), designed to perform low-cost, simple, and rapid indoor coordinate measurements of large-sized objects exploiting the principles of photogrammetry. A preliminary uncertainty assessment referring to the measured point coordinates in the 3D space is presented as well, focusing on the sources of measurement uncertainty and the related propagation laws.

Compared to other existing systems, the novelty of MScMS-II is mainly related to its technical and operational characteristics, which are: scalability, i.e. the capability to extend the measurement domain in order to cover large and geometrically complex working volumes 




Fig. 1. MScMS-II architecture [8]. The dashed lines represent visual links between sensor nodes and retro-reflective spheres (indicated as A and B) equipping the hand-held probe. The Bluetooth connection is established between each node and the processing system.

by properly distributing the network sensors; all-around visibility of the measuring probe; wireless connection of network sensors; layout optimisation, intended as the capability to automatically suggest the optimal sensor positions in order to efficiently cover a given working volume; measurement uncertainty automatically calculated and indicated for every measurement; cooperation of blocks of sensors in order to optimise point acquisitions, system auto-diagnostics, and power consumption; sensor fusion, which is the capability to integrate the metrological system with other spatially distributed sensors (of temperature, humidity, vibrations, light intensity, etc.) in order to provide environmental mapping of the working volume and monitor the operating conditions of the system for auto-diagnostics or self-calibration.

\section{System description}

The MScMS-II consists of three basic units (Fig. 1) [8]: a network ("constellation") of wireless sensor devices, suitably distributed all around the measurement volume, to estimate $3 \mathrm{D}$ coordinates of reflective markers; a mobile wireless and hand-held probe, equipped with two reflective markers, to "touch" the measurement points; a dataprocessing system, using Bluetooth connection, to acquire and elaborate data sent by each network node.

An earlier prototype of MScMS exploited UltraSound (US) transceivers in order to communicate and evaluate mutual distances between the network nodes and the hand-held probe [5]. The poor characteristics of US devices (non-punctiform dimensions, speed of sound dependence on operating temperature, reflection and diffraction of US signal, etc.) caused a low accuracy in the measurement results [5]. In order to enhance system performance, current version makes use of IR-based optical sensors (IR cameras), estimating the position of passive

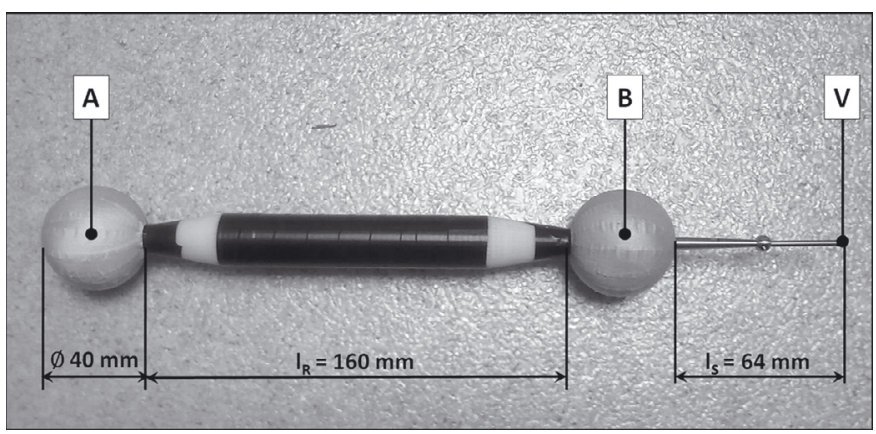

Fig. 2. Mobile measuring probe [8]. A and $B$ are the two spherical retro-reflective markers and $\mathrm{V}$ is the tip.

retro-reflective markers from their projections in different camera views.

Currently, a prototype of the distributed network has been set up by using commercial low-cost IR cameras, characterized by an interpolated resolution of $1024 \times$ 768 pixels (native resolution is $128 \times 96$ pixels), a maximum sample rate of $100 \mathrm{~Hz}$, and a Field Of View (FOV) of approximately $40^{\circ} \times 30^{\circ}$. Each camera implements a real-time multi-object tracking engine, allowing to track up to four IR light sources (IR spots). In order to work with passive markers, each camera was coupled with a near-IR light source, consisting of a 160-chip LED array with a peak wavelength of $940 \mathrm{~nm}$ and an half-Field Of View of approximately $80^{\circ}$. The overall sensor set (camera and LED array) weights about $500 \mathrm{~g}$ and is $13 \times 13 \times 15 \mathrm{~cm}$ sized.

Given a fixed number of cameras, all operating conditions being unchanged, the actual working volume, intended as the region within which the spatial position of a single marker can be reconstructed, depends on the technical specifications of IR cameras (e.g. FOV, sensitivity, pixel resolution and focal length) and light sources (e.g. LED power and wave length), as well as on their relative position and orientation [9], and on the size of the markers. According to triangulation principles, this volume consists of the volume of intersection of the "field-of-sensing" of at least two cameras.

The mobile probe (Fig. 2) consists of a rod, equipped with two reflective markers at the extremes and a stick at one end to physically "touch" the measurement points. The markers have been made by wrapping around polystyrene spheres a retro-reflective silver transfer film.

Referring to Figure 2, as the probe tip (V) lies on the same line of the centres of markers (A, B), spatial coordinates of point $\boldsymbol{x}_{V} \equiv\left(x_{V}, y_{V}, z_{V}\right)$ can be univocally determined by the following linear equation:

$$
\boldsymbol{x}_{V}=\boldsymbol{x}_{A}+\frac{\left(\boldsymbol{x}_{B}-\boldsymbol{x}_{A}\right)}{\left\|\boldsymbol{x}_{B}-\boldsymbol{x}_{A}\right\|} d_{V-A}
$$

where $\boldsymbol{x}_{A} \equiv\left(x_{A}, y_{A}, z_{A}\right)$ and $\boldsymbol{x}_{B} \equiv\left(x_{B}, y_{B}, z_{B}\right)$ identify the centres of markers $A$ and $B$, respectively. The term

$$
d_{V-A}=\left\|\boldsymbol{x}_{V}-\boldsymbol{x}_{A}\right\|
$$

is a priori known as it depends on probe geometry. 


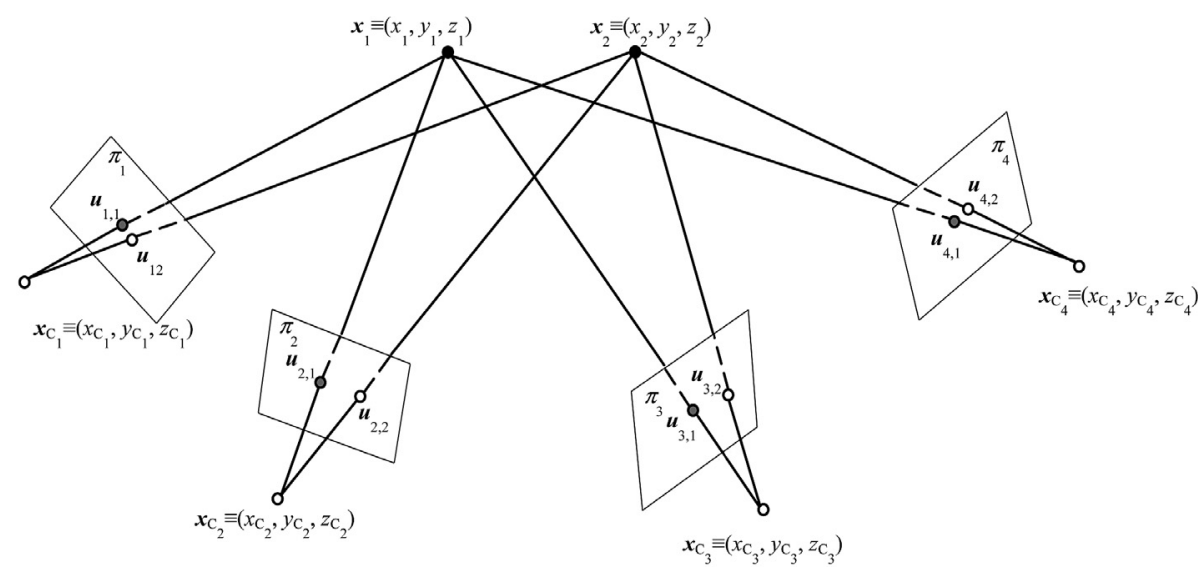

Fig. 3. Graphical representation of the localization problem when a setup of four cameras $\left(n_{c}=4\right)$ is used to reconstruct the $3 \mathrm{D}$ position of two markers $(m=2)$.

The so obtained values of $\boldsymbol{x}_{V} \equiv\left(x_{V}, y_{V}, z_{V}\right)$ must be further corrected by the error due to the non-punctiform shape of the tip. To this end a specific algorithm has been implemented in the MScMS-II software.

The probe has been calibrated at nominal temperature $T=21{ }^{\circ} \mathrm{C}$ and relative humidity $R H=27 \%$ using a coordinate-measuring machine (DEA IOTA 0101).

The data processing hardware consists of a $2.5 \mathrm{GHz}$ computer platform, connected to a set of IR cameras via a Bluetooth link. Each camera provides the data processing system with the $2 \mathrm{D}$ coordinates of the IR spot(s) in its view plane, thus saving computational effort to the computer platform that does not have to perform any image analysis or identification of spot coordinates.

According to the hardware-software configuration, a maximum number of seven IR sensors sequentially sampled can be managed by a single PC unit. A modular approach, based on multiple processing units sharing the information of different camera sets, can be implemented to enlarge the working volumes.

The processing software implements: layout evaluation, designing and analysing sensor network configurations; system calibration, providing position, orientation and technical parameters of sensors; 3D point localization; and data elaboration procedures [8].

\section{Localization algorithm}

In general, given the $3 \mathrm{D}$ coordinates of a point $\boldsymbol{x}_{j} \equiv$ $\left(x_{j}, y_{j}, z_{j}\right)$, its $2 \mathrm{D}$ position $\boldsymbol{u}_{i j} \equiv\left(u_{i j}, v_{i j}\right)$ in different camera image planes $\pi_{i}$ (Fig. 3) can be obtained through the following relationship (perspective transformation) [7]:

$$
\boldsymbol{a}_{i j}=\boldsymbol{P}_{i} \boldsymbol{X}_{j}
$$

where $\boldsymbol{a}_{i j} \equiv\left[a_{i j} b_{i j} w_{i j}\right]^{T}=w_{i j}\left[\begin{array}{lll}u_{i j} & v_{i j} & 1\end{array}\right]^{T}$ is the $2 \mathrm{D}$ pixel position in homogeneous coordinates, $\boldsymbol{X}_{j} \equiv$ $\left[\begin{array}{llll}x_{j} & y_{j} & z_{j} & 1\end{array}\right]^{T}$ is the corresponding $3 \mathrm{D}$ position in homogeneous coordinates, and $\boldsymbol{P}_{i} \in \mathbb{R}^{3 \times 4}$ is the projection matrix of the $i$ th camera. It contains 6 external parameters describing camera position and orientation (3D position coordinates of the camera perspective centre $\boldsymbol{x}_{C j} \equiv\left(x_{C j}, y_{C j}, z_{C j}\right)$ and orientation angles of its optical axis $\left(\omega_{i}, \phi_{i}\right.$ and $\left.\kappa_{i}\right)$, and 5 internal parameters which describe the inner properties of the camera (principal distance $c_{i}$, principal point coordinates $\left(u_{0 i}, v_{0 i}\right)$, angle between axes $\vartheta_{i}$, and scale coefficient of axes $k_{i}$ ) [7].

Each projection matrix $\boldsymbol{P}_{i} \in \mathbb{R}^{3 \times 4}$ can be obtained as the product of a non-zero scale factor $\mu_{i}$ with the matrix of internal parameters $\boldsymbol{K}_{i} \in \mathbb{R}^{3 \times 4}$ and the exterior orientation matrix $\boldsymbol{M}_{i} \in \mathbb{R}^{4 \times 4}[7]$ :

$$
\boldsymbol{P}_{i}=\mu_{i} \boldsymbol{K}_{i} \boldsymbol{M}_{i} .
$$

Matrix $\boldsymbol{M}_{i}$ incorporates a shift of axes origin of 3D point space into the camera centre coordinates rotated into the camera coordinate system, and a rotation according to $\boldsymbol{R}_{i} \in \mathbb{R}^{3 \times 3}$, which is the rotation matrix obtained by considering three sequential rotations corresponding to the orientation angles of camera optical axis $\left(\omega_{i}\right.$ about the $X$ axis, $\phi_{i}$ about the once-rotated $Y$-axis, and $\kappa_{i}$ about the twice-rotated $Z$-axis).

From the operational point of view, the elements of $\boldsymbol{P}_{i}$ are directly obtained by the system calibration procedure $[8,10]$.

According to equation (4), the 11 physical parameters of each camera can be derived from the element of $\boldsymbol{P}_{i}$ by simple mathematical transformations [7].

In general, the so called collinearity equations derive from equation (3). These equations are the basis of photogrammetry theory and originate from the perspective projection of a point in the $3 \mathrm{D}$ space onto a given camera viewing plane $[6,7]$ :

$$
\begin{aligned}
& u_{i j}=u_{0 i}-c_{u i} \\
& \times \frac{r_{11 i}\left(x_{j}-x_{C i}\right)+r_{12 i}\left(y_{j}-y_{C i}\right)+r_{13 i}\left(z_{j}-z_{C i}\right)}{r_{31 i}\left(x_{j}-x_{C i}\right)+r_{32 i}\left(y_{j}-y_{C i}\right)+r_{33 i}\left(z_{j}-z_{C i}\right)}-\delta u_{i} \\
& v_{i j}=v_{0 i}-c_{v i} \\
& \times \frac{r_{21 i}\left(x_{j}-x_{C i}\right)+r_{22 i}\left(y_{j}-y_{C i}\right)+r_{23 i}\left(z_{j}-z_{C i}\right)}{r_{31 i}\left(x_{j}-x_{C i}\right)+r_{32 i}\left(y_{j}-y_{C i}\right)+r_{33 i}\left(z_{j}-z_{C i}\right)}-\delta v_{i}
\end{aligned}
$$


where $c_{u i}$ and $c_{v i}$ are given by the principal distance (focal length) $c_{i}$ scaled by different factors respectively in the $u$ and $v$ directions, $\delta u_{i}$ and $\delta v_{i}$ are the total lens distortions respectively in the $u$ and $v$ directions, and $r_{11}, \ldots, r_{33}$ are the elements of the rotation matrix $\boldsymbol{R}_{i}$.

According to the collinearity equations, given a camera layout (i.e. $n_{c}$ cameras, with known external and internal parameters) focused on $m$ markers, for each $m$-uple of $2 \mathrm{D}$ pixel coordinates $\boldsymbol{u}_{i j} \equiv\left(u_{i j}, v_{i j}\right)$, with $i=1, \ldots, n(2 \leqslant n \leqslant$ $n_{c}$ ) and $j=1, \ldots, m$, the localization algorithm provides the $3 \mathrm{D}$ coordinates of the corresponding $m$ retro-reflective markers (Fig. 3).

The localization procedure is articulated in two main steps:

1) find the correspondences among $2 \mathrm{D}$ points in different image views,

2) match the $2 \mathrm{D}$ information of different camera views for recovering the spatial coordinates of the $3 \mathrm{D}$ point.

\subsection{Finding of correspondences among 2D points in different image views}

As to the first step, epipolar geometry, i.e. the intrinsic projective geometry between two views, has been used to correlate information from multiple camera images $[6,7]$. The correlation between the $2 \mathrm{D}$ pixel coordinates of two points, $\boldsymbol{u} \equiv(u, v)$ and $\boldsymbol{u}^{\prime} \equiv\left(u^{\prime}, v^{\prime}\right)$, detected by two different cameras, states to what extent they can be considered as the projections of the same $3 \mathrm{D}$ point onto the camera image planes. According to epipolar geometry principles, given a pair of images, to a generic point $\boldsymbol{u} \equiv(u, v)$ in the first image corresponds a line $\ell$ (i.e. the epipolar line) in the second image (Fig. 4). The epipolar line is defined as the intersection of the image plane of the second camera with the plane passing through point $\boldsymbol{u} \equiv(u, v)$ and the two camera centres, $\boldsymbol{x}_{C_{1}}$ and $\boldsymbol{x}_{C_{2}}$, which will also contain the reconstructed $3 \mathrm{D}$ point $\boldsymbol{x} \equiv(x, y, z)$. This line can be drawn whenever the projection matrices of the given pair of cameras are known. The image view of the 3D point in the second image $\boldsymbol{u}^{\prime} \equiv\left(u^{\prime}, v^{\prime}\right)$ will thus lie on the epipolar line $\ell$. As a matter of fact, the point correspondence can be found by evaluating the distance between the $2 \mathrm{D}$ pixel $\boldsymbol{u}^{\prime} \equiv\left(u^{\prime}, v^{\prime}\right)$ in the second image and the epipolar line corresponding to the $2 \mathrm{D}$ pixel $\boldsymbol{u} \equiv(u, v)$ in the first image.

The epipolar line corresponding to point $\boldsymbol{u} \equiv(u, v)$ can be drawn through the Fundamental Matrix, i.e. the unique matrix $\boldsymbol{F} \in \mathbb{R}^{3 \times 3}$ which, for all the corresponding points $\boldsymbol{u} \leftrightarrow \boldsymbol{u}^{\prime}$, satisfies:

$$
\boldsymbol{a}^{T} \boldsymbol{F} \boldsymbol{a}^{\prime}=0
$$

where $\boldsymbol{a} \equiv\left[\begin{array}{lll}a & b & w\end{array}\right]^{T}=w\left[\begin{array}{lll}u & v & 1\end{array}\right]^{T}$ and $\boldsymbol{a}^{\prime} \equiv$ $\left[\begin{array}{lll}a^{\prime} & b^{\prime} & w^{\prime}\end{array}\right]^{T}=w^{\prime}\left[\begin{array}{ll}u^{\prime} & v^{\prime}\end{array} 1\right]^{T}$ are the $2 \mathrm{D}$ pixel positions of $\boldsymbol{u} \equiv(u, v)$ and $\boldsymbol{u}^{\prime} \equiv\left(u^{\prime}, v^{\prime}\right)$ expressed in homogeneous coordinates.

For each pair of cameras the fundamental matrix can be computed according to their projection matrices [7].

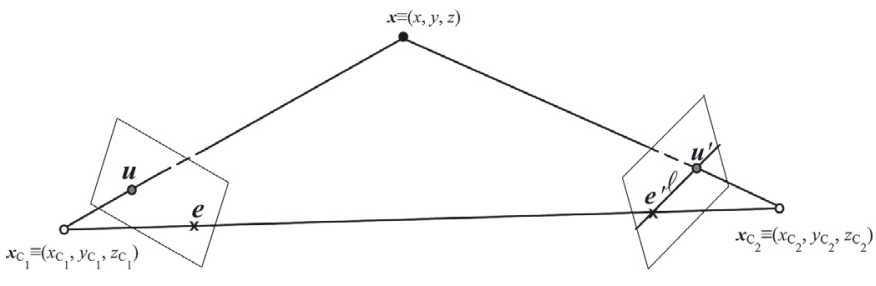

Fig. 4. Epipolar geometry principles. $\boldsymbol{x}_{C_{1}}$ and $\boldsymbol{x}_{C_{2}}$ are the camera centres, $\boldsymbol{x} \equiv(x, y, z)$ is the $3 \mathrm{D}$ point. $\boldsymbol{u} \equiv(u, v)$ and $\boldsymbol{u}^{\prime} \equiv\left(u^{\prime}, v^{\prime}\right)$ represent the $2 \mathrm{D}$ projection of $\boldsymbol{x}$ onto the image planes of the first and second camera respectively. $e$ and $\boldsymbol{e}^{\prime}$ are the epipoles of the two cameras, i.e. the intersection of the line joining the two camera centres with the image plane. $\ell$ is the epipolar line corresponding to point $\boldsymbol{u} \equiv(u, v)$.

As the epipolar distance is proportional to the reprojection error after triangulation, large epipolar distances mean pixel correlation mismatches and large reprojection errors. A threshold method has been implemented to find correspondences between different image views. Therefore, the association between point $\boldsymbol{u} \equiv(u, v)$ in the first camera view and point $\boldsymbol{u}^{\prime} \equiv\left(u^{\prime}, v^{\prime}\right)$ in the second camera view is verified by applying the following threshold constraint:

$$
\boldsymbol{a}^{T} \boldsymbol{F} \boldsymbol{a}^{\prime}<\varepsilon
$$

where $\varepsilon$ is an empirically-defined threshold according to camera resolution.

This procedure must be carried out for each couple of cameras in the measurement layout.

The concurrent presence of more than one retroreflective marker within the working volume could give rise to some ambiguities in measurement point recovery. In some practical cases, probe positioning with respect to the IR sensor and its orientation could correspond to a very small distance between the two pixels in an image view. In order to reduce the errors in pixel correlation, a minimum search approach has been implemented. Stated that two pixels $\boldsymbol{u}^{\prime} \equiv\left(u^{\prime}, v^{\prime}\right)$ and $\boldsymbol{u}^{\prime \prime} \equiv\left(u^{\prime \prime}, v^{\prime \prime}\right)$ in the second camera view verified the threshold constraint (Eq. (7)), the point $\boldsymbol{u} \equiv(u, v)$ in the first camera view will be correlated to the one $\left(\boldsymbol{u}^{*} \equiv\left(u^{*}, v^{*}\right)\right)$ having the minimum distance to its epipolar line $\ell$ :

$$
\boldsymbol{a}^{*}: \boldsymbol{a} \boldsymbol{F} \boldsymbol{a}^{*}=\min \left(\boldsymbol{a} \boldsymbol{F} \boldsymbol{a}^{\prime}, \boldsymbol{a} \boldsymbol{F} \boldsymbol{a}^{\prime \prime}\right) .
$$

\subsection{D point recovering}

The second step of the localization algorithm deals with the triangulation problem [6,7]. Given its $2 \mathrm{D}$ positions in $n$ different image planes (with $2 \leqslant n \leqslant n_{c}$ ), the 3D coordinates of a point $\boldsymbol{x} \equiv(x, y, z)$ can be obtained by intersecting the camera projection lines (triangulation).

An approach based on the linearization of the collinearity equations (Eq. (5)) has been implemented. This method is applied in most localization algorithms in photogrammetry for three main reasons: its implementation facilitates the development of efficient algorithms for 


$$
\begin{aligned}
& {\left[\begin{array}{lll}
\dot{\boldsymbol{B}}^{T} \boldsymbol{W} \dot{\boldsymbol{B}}+\dot{\boldsymbol{C}}^{T} \boldsymbol{W}_{C} \dot{\boldsymbol{C}}+\dot{\boldsymbol{W}} & \dot{\boldsymbol{B}}^{T} \boldsymbol{W} \hat{\boldsymbol{B}}+\dot{\boldsymbol{C}}^{T} \boldsymbol{W}_{C} \hat{\boldsymbol{C}} & \dot{\boldsymbol{B}}^{T} \boldsymbol{W} \ddot{\boldsymbol{B}}+\dot{\boldsymbol{C}}^{T} \boldsymbol{W}_{C} \ddot{\boldsymbol{C}} \\
\left(\dot{\boldsymbol{B}}^{T} \boldsymbol{W} \hat{\boldsymbol{B}}+\dot{\boldsymbol{C}}^{T} \boldsymbol{W}_{C} \hat{\boldsymbol{C}}\right)^{T} & \hat{\boldsymbol{B}}^{T} \boldsymbol{W} \hat{\boldsymbol{B}}+\hat{\boldsymbol{C}}^{T} \boldsymbol{W}_{C} \hat{\boldsymbol{C}}+\hat{\boldsymbol{W}} & \hat{\boldsymbol{B}}^{T} \boldsymbol{W} \ddot{\boldsymbol{B}}+\hat{\boldsymbol{C}}^{T} \boldsymbol{W}_{C} \ddot{\boldsymbol{C}} \\
\left(\dot{\boldsymbol{B}}^{T} \boldsymbol{W} \ddot{\boldsymbol{B}}+\dot{\boldsymbol{C}}^{T} \boldsymbol{W}_{C} \ddot{\boldsymbol{C}}\right)^{T} & \left(\tilde{\boldsymbol{B}}^{T} \boldsymbol{W} \tilde{\boldsymbol{B}}+\tilde{\boldsymbol{C}}^{T} \boldsymbol{W}_{C} \tilde{\boldsymbol{C}}\right)^{T} & \ddot{\boldsymbol{B}}^{T} \boldsymbol{W} \ddot{\boldsymbol{B}}+\ddot{\boldsymbol{C}}^{T} \boldsymbol{W}_{C} \ddot{\boldsymbol{C}}+\ddot{\boldsymbol{W}}
\end{array}\right]\left[\begin{array}{c}
\dot{\boldsymbol{\delta}} \\
\hat{\boldsymbol{\delta}} \\
\ddot{\boldsymbol{\delta}}
\end{array}\right]} \\
& =\left[\begin{array}{l}
\dot{\boldsymbol{B}}^{T} \boldsymbol{W} \boldsymbol{f}+\dot{\boldsymbol{C}}^{T} \boldsymbol{W}_{C} \boldsymbol{f}_{C}-\boldsymbol{W} \dot{\boldsymbol{f}} \\
\hat{\boldsymbol{B}}^{T} \boldsymbol{W} \boldsymbol{f}+\hat{\boldsymbol{C}}^{T} \boldsymbol{W}_{C} \boldsymbol{f}_{C}-\boldsymbol{W} \hat{\boldsymbol{f}} \\
\ddot{\boldsymbol{B}}^{T} \boldsymbol{W} \boldsymbol{f}+\ddot{\boldsymbol{C}}^{T} \boldsymbol{W}_{C} \boldsymbol{f}_{C}-\boldsymbol{W} \boldsymbol{f}
\end{array}\right]
\end{aligned}
$$

formation and solution of the normal equations in leastsquares adjustment; each observable (known variable) in equation 5 may be weighted in least-squares adjustment according to the related covariance matrix; it makes the computation of the uncertainty of $\boldsymbol{x}$ coordinates automatic [7].

As a matter of fact, the 3D position coordinates of a point $\boldsymbol{x} \equiv(x, y, z)$ are reconstructed from $n$ camera image points by intersecting their viewing rays (see Figs. 3 and 4 ). This involves solving the $2 \times n$ collinearity equations for $\boldsymbol{x}$. The result is an overdetermined (non-linear) equation system.

For each camera and each point, equation (5) can be reorganized into the following form:

$$
\begin{aligned}
& \left(u-u_{0}\right)+c_{u} \\
& \times \frac{r_{11}\left(x-x_{C}\right)+r_{12}\left(y-y_{C}\right)+r_{13}\left(z-z_{C}\right)}{r_{31}\left(x-x_{C}\right)+r_{32}\left(y-y_{C}\right)+r_{33}\left(z-z_{C}\right)}+\delta u=f_{1} \\
& \left(v-v_{0}\right)+c_{v} \\
& \quad \times \frac{r_{21}\left(x-x_{C}\right)+r_{22}\left(y-y_{C}\right)+r_{23}\left(z-z_{C}\right)}{r_{31}\left(x-x_{C}\right)+r_{32}\left(y-y_{C}\right)+r_{33}\left(z-z_{C}\right)}+\delta v=f_{2}
\end{aligned}
$$

where (observations) $f_{1}$ and $f_{2}$ are the differences between the measured and the computed $2 \mathrm{D}$ coordinates.

Furthermore, there are many cases in which other parameters or equations must be added to the solution. For example, it might be necessary to incorporate known geometric constraints, or determine additional calibration parameters for the camera. Considering all these constraints and additional parameters, the general linearized expression of the collinearity equations associated with the geometric constraints equations can thus be written as [7]:

$$
\begin{aligned}
& v+\dot{\boldsymbol{B}} \dot{\boldsymbol{\delta}}+\hat{\boldsymbol{B}} \hat{\boldsymbol{\delta}}+\ddot{B} \ddot{\boldsymbol{\delta}}=f \\
& \boldsymbol{v}_{C}+\dot{\boldsymbol{C}} \dot{\boldsymbol{\delta}}+\hat{\boldsymbol{C}} \hat{\boldsymbol{\delta}}+\ddot{\boldsymbol{C}} \ddot{\boldsymbol{\delta}}=\boldsymbol{f}_{C}
\end{aligned}
$$

where $\boldsymbol{f}$ is the vector of observations obtained by computing equation (9) for the approximations for the 6 external camera, the 5 internal camera parameters and added parameters, and the 3 point coordinates in the 3D space, and $\boldsymbol{v}$ is the vector of the residuals of point coordinates in the 2D image plane of the camera. The unknowns in equations (10a) and (10b) are the corrections to the approximations for the 6 external camera parameters (i.e. the elements of $\dot{\boldsymbol{\delta}}=$ $\left.\left[x_{C}-x_{C 0} y_{C}-y_{C 0} z_{C}-z_{C 0} \omega-\omega_{0} \phi-\phi_{0} \kappa-\kappa_{0}\right]^{T}\right)$, the corrections to the approximations for the 5 internal camera parameters, as well as other additional parameters and constraints (i.e. the elements of $\left.\hat{\boldsymbol{\delta}}=\left[u_{0}-u_{00} v_{0}-v_{00} c-c_{0} k-k_{0} \vartheta-\vartheta_{0} \ldots \ldots\right]^{T}\right)$, and the corrections to the approximations for the 3 point coordinates in the $3 \mathrm{D}$ space (i.e. the elements of $\left.\ddot{\boldsymbol{\delta}}=\left[\begin{array}{lll}x-x_{0} & y-y_{0} z-z_{0}\end{array}\right]^{T}\right) . \dot{\boldsymbol{B}} \in \mathbb{R}^{2 \times 6}$ is the matrix of partial derivatives of the two functions in equation (9) with respect to each of the 6 external camera parameters, $\hat{\boldsymbol{B}} \in \mathbb{R}^{2 \times n^{\prime}}$ (where $n^{\prime} \geqslant 5$ ) is the matrix of partial derivatives of the two functions in equation (9) with respect to each of the 5 internal camera parameters and added parameters, $\ddot{\boldsymbol{B}} \in \mathbb{R}^{2 \times 3}$ is the matrix of partial derivatives of the two functions in equation (9) with respect to each of the $3 \mathrm{D}$ point coordinates.

Equation (10b) is the set of linearized equations of geometric constraints on the same parameters.

According to equations (10a) and (10b), the leastsquare normal equations associated with one image of one point are:

$$
\text { See equation (11) above. }
$$

where $\boldsymbol{W} \in \mathbb{R}^{2 \times 2}$ is the weight matrix (i.e. the inverse of the covariance matrix) associated with the point coordinates $(u, v)$ in the $2 \mathrm{D}$ camera image (see Sect. 4.1). In the same way, matrix $\dot{\boldsymbol{W}}, \hat{\boldsymbol{W}}$, and $\ddot{\boldsymbol{W}}$ refer to $\dot{\boldsymbol{\delta}}, \hat{\boldsymbol{\delta}}$, and $\ddot{\boldsymbol{\delta}}$ unknown vectors. In equation (11) a compact notation $(\dot{\boldsymbol{f}}$, $\hat{\boldsymbol{f}}, \ddot{\boldsymbol{f}})$ has been used in order to indicate the components of vector $\boldsymbol{f}$ referring to $\dot{\boldsymbol{\delta}}, \hat{\boldsymbol{\delta}}$, and $\ddot{\boldsymbol{\delta}}$ respectively.

Equation (11) can be rewritten as:

$$
\left[\begin{array}{lll}
\dot{N}^{T} & \widetilde{N} & \overline{\boldsymbol{N}} \\
\bar{N}^{T} & \hat{N} & \tilde{N} \\
\bar{N}^{T} & \tilde{N}^{T} & \ddot{\boldsymbol{N}}
\end{array}\right]\left[\begin{array}{l}
\dot{\delta} \\
\hat{\boldsymbol{\delta}} \\
\ddot{\boldsymbol{\delta}}
\end{array}\right]=\left[\begin{array}{l}
\dot{\boldsymbol{t}} \\
\hat{\boldsymbol{t}} \\
\ddot{\boldsymbol{t}}
\end{array}\right] .
$$

Assuming that all systematic errors have been removed (this issue is seldom completely true in practice and it is addressed by adding correction parameters), it is possible to assert that the uncertainty in each point image coordinates is uncorrelated with that of any other image coordinates, both other points imaged on the same image and other images of the same point, although uncertainty in the $u$ and $v$ coordinates of any single image point may be correlated. This assumption implies that the total covariance $\boldsymbol{\Sigma}$ matrix and weight $\boldsymbol{W}$ matrix, containing the covariance and the weight information for all the image coordinates, are block diagonal. Each block is a $2 \times 2$ matrix corresponding to a single set of image measurements for one point. 
Extending equations (11) and (12) to a set of $n$ cameras and $m$ points, the obtained normal equations maintains the same formulation and have a regular structure which can be easily implemented in a numerical routine [7]. The same holds for equations (10a) and (10b).

The localization algorithm used in MScMS-II implements a simplified adaptation of equations (10a) and (10b), in which internal and external camera parameters are known (from self-calibration procedure), and for each measuring probe a geometrical constraint (i.e. the distance between the two probe markers) is fixed. This entails the use of at least two cameras viewing the two probe markers at the same time.

A set of filtering algorithms has been implemented as well, in order to remove possible noise or falsemeasurements (external IR sources, reflections, etc.), and distinguish between the two probe markers.

Furthermore, it has to be noted that, as the triangulation results are based on the 2D image views of different cameras, they are strongly affected by camera synchronization issues. As a matter of fact, the 3D point reconstruction algorithm should use the $2 \mathrm{D}$ position coordinates of the same point as seen by the different cameras at the same time instant (synchronized camera sampling). Whenever a sequential sampling procedure is implemented, the higher the number of cameras the higher the total acquisition delay and thus possible discrepancies among different image views. Whereas it could represent a problem for tracking dynamic objects, sequential sampling has negligible influence on measurement performance as data acquisition is made by keeping the probe in static conditions.

Multi-camera calibration problem is faced by using a fully automatic single-point self-calibration technique able to reconstruct internal parameters as well as positions and orientations (external parameters) of a camera set $[8,10]$.

As the external camera parameters are provided in an unknown coordinate reference system, having the origin in the centre of the cloud of points, a further step for aligning and scaling the world coordinate system is needed. To this end, a laser cut aluminium square $(300 \times 400 \mathrm{~mm}$ sized $)$, calibrated to submillimeter accuracy at nominal temperature $T=21^{\circ} \mathrm{C}$ and relative humidity $R H=27 \%$ using a coordinate-measuring machine (DEA IOTA 0101), is used as reference artefact [8].

Calibration procedure yields 5 internal camera parameters, i.e. the elements of matrix $\boldsymbol{K}$ in equation (4) (the same for all cameras), and 6 external camera parameters, i.e. the elements of matrix $\boldsymbol{R}$ in equation (4), as well as their respective covariance matrices $\boldsymbol{\Sigma}_{\boldsymbol{K}}$ and $\boldsymbol{\Sigma}_{\boldsymbol{R}}$, under the hypothesis of no correlation between the two sets of parameters.

\section{Uncertainty evaluation}

In order to evaluate the metrological potentiality of the system, a preliminary uncertainty evaluation of measured $3 \mathrm{D}$ point coordinates $(x, y, z)$ has been performed using the Multivariate Law of Propagation of Uncertainty
(MLPU) [11]. Various approaches are suggested in scientific literature according to the different problems at hand $[3,6,7]$. An alternative approach to MLPU could be based on Monte Carlo Sampling technique which has the potential advantage of being independent of linearization [3].

In the present work the MLUP has been preferred for two reasons at least. For LSM applications, the sensor data are typically accurate to one part in $10^{4}$ or better, so that non-linearity in the models do not have a significant effect on the uncertainty estimates. On the other hand, uncertainty assessment in the photogrammetry approach is based on the uncertainty propagation within the leastsquare adjustment process of variances and covariances of input estimated parameters (see Eqs. (10a) and (10b)).

The main contributions to overall uncertainty of $3 \mathrm{D}$ point coordinates may be traced in the following issue:

1) uncertainty of $2 D$ point coordinates, which refers to the $2 \mathrm{D}$ pixel coordinates of point projection in the image plane,

2) uncertainty of camera calibration parameters, which is associated with the internal and external camera parameters obtained in the calibration phase,

3) camera synchronization error, which is considered negligible in static conditions (consideration would be necessary for a dynamic approach, i.e. in case of point tracking),

4) uncertainty of $3 D$ point coordinates, which can be traced back to the triangulation algorithm for $3 \mathrm{D}$ point reconstruction,

5) uncertainty of probe tip coordinates, which actually determines the uncertainty of the point coordinates measured by the MScMS-II.

Hereinafter, the uncertainties of coordinates referring to $2 \mathrm{D}$ image, $3 \mathrm{D}$ point and probe tip are discussed and analyzed in more detail.

\subsection{Uncertainty of 2D point coordinates}

Assuming that the uncertainty in each point image coordinate is uncorrelated with that of any other image coordinate (this is true in absence of systematic errors), both other points imaged on the same image and other images of the same point, only uncertainty in the $u$ and $v$ coordinates of any single image point may be correlated. Hence, each couple of coordinates $\left(u_{i j}, v_{i j}\right)$ in the $2 \mathrm{D}$ image plane of a given camera $i$, corresponding to a specific point $j$ in the $3 \mathrm{D}$ space, can be associated with a covariance matrix $\Sigma_{i j} \in \mathbb{R}^{2 \times 2}$.

According to the MScMS-II working principles, the centres of two spherical markers must be localized (Fig. 1). In this case, the main factors that may contribute to form $\boldsymbol{\Sigma}_{i j}$ matrix are: camera technical characteristics (resolution, focal length, FOV, sensitivity, lens distortion), system layout geometry (size of markers, distance between camera and markers, position of markers with respect to the normal of the camera plane of view, fraction of marker 
surface visible by each camera), computational procedures (2D point correspondence analysis, image processing algorithms), and noise or false-measurements (camera vibrations, external IR sources, reflections, etc.).

All these factors give their direct or indirect contribution to the uncertainty in the measurement of $2 \mathrm{D}$ point coordinates. In general, considering the image plane of a camera, the average measurement uncertainty of nonsignalized points is around $0.2 \div 0.5$ pixel [6]. If the feature to be measured consists of a symmetrical distribution of pixel values, many mathematical methods can be implemented to determine the centre, among these worth mentioning: the Local Centroid method, the Correlation method, the Least-Squares Matching method, and the Structural methods [6]. All of them may be efficiently implemented in order to obtain centre point coordinates and the corresponding uncertainty. The Least-Squares Matching method, however, is the most convenient since it automatically provides the covariance matrix of the two computed coordinates.

A significant factor in determining $2 \mathrm{D}$ point uncertainty is the size (diameter) of imaged points. It is directly correlated to marker position and distance, and camera characteristics (resolution, focal length, FOV, sensitivity and lens distortion). The optimum marker size ranges between about 5 and 15 pixels in diameter [6]. Smaller points do not provide enough information, while larger points diameters may result in too large numbers of observations to be processed. An empirical relationship between marker size and achievable point uncertainty reported by [6] shows that as the marker size increases over 25 pixels, the function rapidly converges to an uncertainty of about 0.005 pixel. On the other hand, with a marker size under 5 pixels, the uncertainty reaches values over 0.01 pixel.

For a preliminary assessment of point coordinate uncertainty in MScMS-II measurements, considering the low-level resolution of the cameras used for the prototype assembling, the pixel uncertainty (intended as standard deviation of each of the two pixel coordinates $(u, v)$ ) has been assumed equal to 0.5 pixel with no covariance between the two coordinates.

In standard operational condition of the system, a MATLAB routine for image processing, based on LeastSquares Matching method, provides the covariance matrices for the centres of each of the two probe markers, as well as the $(u, v)$ coordinates.

\subsection{Uncertainty of 3D point coordinates}

According to equation (12) extended to a set of $n$ cameras and $m$ points, the following reduced normal equation may be derived:

$$
\ddot{\boldsymbol{\delta}}=\ddot{\boldsymbol{N}}^{-1}\left(\ddot{\boldsymbol{t}}-\overline{\boldsymbol{N}}^{T} \dot{\boldsymbol{\delta}}-\tilde{\boldsymbol{N}}^{T} \hat{\boldsymbol{\delta}}\right)
$$

Once the covariances $\dot{\boldsymbol{\Sigma}}$ and $\hat{\boldsymbol{\Sigma}}$ of the $\dot{\boldsymbol{\delta}}$ and $\hat{\boldsymbol{\delta}}$ parameters are known (by the calibration procedure), the covariance $\ddot{\boldsymbol{\Sigma}}$ of the point coordinates ( $\ddot{\boldsymbol{\delta}}$ parameters) can be calculated by applying the MLPU to equation (13). It can be demonstrated that the propagated point covariance is given by $[7]$ :

$$
\ddot{\boldsymbol{\Sigma}}=\ddot{\boldsymbol{N}}^{-1}+\ddot{\boldsymbol{N}}^{-1} \overline{\boldsymbol{N}}^{T} \dot{\boldsymbol{\Sigma}} \overline{\mathbf{N}} \ddot{\boldsymbol{N}}^{-1}+\ddot{\boldsymbol{N}}^{-1} \tilde{\boldsymbol{N}}^{T} \hat{\boldsymbol{\Sigma}} \tilde{\boldsymbol{N}} \ddot{\boldsymbol{N}}^{-1} \text {. }
$$

The diagonal $3 \times 3$ block elements of $\ddot{\boldsymbol{\Sigma}}$ are the covariances of the $3 \mathrm{D}$ coordinates of measured points.

It must be noted that the covariance of $2 \mathrm{D}$ pixel coordinates is implicitly present in equation (14) (see the definition of $\ddot{N}$ in Eqs. (11) and (12)).

\subsection{Uncertainty of probe tip coordinates}

The covariance of the probe tip coordinates $\boldsymbol{\Sigma}_{V} \in \mathbb{R}^{3 \times 3}$ in each measurement is calculated by applying the MLPU to the linearized form of equation (1), as well:

$$
\boldsymbol{\Sigma}_{V}=\Omega \boldsymbol{\Sigma}_{P} \boldsymbol{\Omega}^{T}
$$

where $\Omega \in \mathbb{R}^{3 \times 7}$ is the Jacobian of the function in equation (1), i.e. the matrix of its partial derivatives with respect to the 3 coordinates of the centres of the two probe markers, and the parameter $d_{V-A}$.

$\boldsymbol{\Sigma}_{P} \in \mathbb{R}^{7 \times 7}$ is the covariance matrix of parameters in equation (1), obtained by extracting from $\ddot{\boldsymbol{\Sigma}}$ the elements corresponding to covariance matrix $\boldsymbol{\Sigma}_{A-B} \in \mathbb{R}^{6 \times 6}$ of $\boldsymbol{x}_{A}$ and $\boldsymbol{x}_{B}$ and adding the variance $\sigma_{d_{V-A}}^{2}$ of $d_{V-A}$ estimated during the probe calibration:

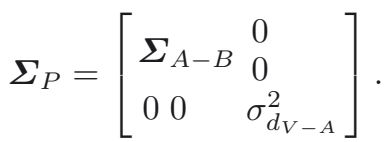

The diagonal elements of $\boldsymbol{\Sigma}_{V}$ are the variances of the 3D coordinates of the point measured by the probe.

They can be used to evaluate the extended uncertainty of point coordinates by extracting the corresponding standard deviations and multiplying them by an opportune coverage factor [11].

The uncertainty contribution due to the correction of the non-punctiform shape of the tip is usually neglected. Therefore, it can be considered by adding minor modifications to equations (15) and (16).

\section{Experimental tests for preliminary metrological characterization}

In the current system configuration, the network layout consists of six commercial low-cost IR sensors, arranged in a $5.0 \times 6.0 \times 3.0 \mathrm{~m}$ working environment according to a grid-based configuration. This results in an actual working volume of about $2.0 \times 2.0 \times 2.0 \mathrm{~m}$.

In Section 4 an approach for preliminary uncertainty evaluation of point coordinates based on the MLPU has been introduced. Assuming for all cameras and measured points a pixel uncertainty (intended as standard deviation of each of the two pixel coordinates $(u, v))$ of 0.5 pixel 
with no covariance between the two coordinates, and a standard deviation of $d_{V-A}$ parameter equal to $0.05 \mathrm{~mm}$, the uncertainty propagation algorithm gives $3 \mathrm{D}$ point coordinate uncertainties in the whole working volume lower than $5 \mathrm{~mm}$.

Performance evaluation, however, requires comparison with independent measurements either from independent control points or through the use of calibrated scale bars spaced throughout the measurement volume. For example, the German guideline VDI/VDE 2634 [12] recommends the use of specific calibrated scale bars that can be employed to determine ISO conformant length measuring errors [3].

Preliminary experimental tests have been carried out to evaluate prototype performance (in terms of measurement accuracy, repeatability, and reproducibility) as well as system potentialities $[8,13]$.

The evaluation of the measurement accuracy of point coordinates has been carried out using the 3D calibrated square of aluminium alloy used for system calibration. The reference points have been measured by the MScMS-II system prototype, by moving the artefact in 5 different positions uniformly distributed within the working volume. The obtained results show that $50 \%$ of the measured points is within a distance of $1.9 \mathrm{~mm}$ from the nominal position, while $94.2 \%$ of results is less than $5 \mathrm{~mm}$ far from the nominal position. At worst the maximum measured distance is below $6.5 \mathrm{~mm}$ [8]. By considering several issues (e.g. geometric distortion of the reconstructed working volume and measurement process) whose effects on measurements strongly depend on the point location within the working volume, the severe experimental testing procedure consistently affect the extent of measurement deviations as well as their high variability.

In a second test, measurement repeatability of point coordinates has been tested on 5 different points, uniformly distributed within the working volume, by repositioning the probe in the same positions for 30 times. It is noteworthy that repeatability characteristics are related to the sensor device performance as well as to the operator skills. Human skills actually represent an external factor related to capabilities in holding the probe in a fixed position. The sample standard deviation of repeatability tests was found to be smaller than $1.25 \mathrm{~mm}$ [8].

Finally, measurement reproducibility of point coordinates has been tested with reference to 5 points, repeating the measurement 30 times with different mobile probe orientations. Reproducibility tests stress the importance on measurement quality of network and probe relative position and orientation. A sample standard deviation smaller than $3.45 \mathrm{~mm}$ has been obtained [8].

\section{Conclusions}

A preliminary assessment of the measurement uncertainty of MScMS-II, a low-cost optical IR-based system for LSM applications, has been presented referring to theoretical calculations and experimental results. The evaluation of the coordinate uncertainty of a measured point in the $3 \mathrm{D}$ space has been performed according to the MLPU, and starting from the triangulation-based localization algorithm and the geometric constraints of the implemented architecture. Further considerations on uncertainties due to self-calibration procedures and sensor data sampling methods have been also included.

The preliminary experimental results, aimed at evaluating the system performance in terms of accuracy, repeatability and reproducibility, reveal MScMS-II limits and potentialities for the application in the field of LSM. As a matter of fact, the system prototype does not appear to be very competitive if compared to commercial systems (e.g. CMMs, laser trackers, iGPS), whose accuracy deviation, in the same working volume, may range from a few micrometers up to one millimetre at worst. On the other hand, it demonstrates a great potential whenever cost and flexibility are considered. While ensuring scalability and modularity that existing commercial systems cannot guarantee, the prototype still has significant room for enhancement mainly related to the improvement of the employed technology. Current CCD sensors $(128 \times 96$ pixels), although very cheap, could be easily replaced with higher performance ones with no much impact on the cost of the entire system, and a significant augmentation in accuracy (one order of magnitude at least).

In a research perspective, a closer examination of factors affecting measurement uncertainty, including IR hardware characteristics, self-calibration and localization algorithms, will be carried out. Further investigation will be devoted to the effects of the calibration and scaling procedures, and to possible correction models for correcting systematic errors. In addition, feasibility of a multiresolution system, integrating the proposed network-based solution with highly accurate optical systems, will be investigated.

\section{References}

1. M.J. Puttock, Large-scale metrology, CIRP Ann. Manuf. Technol. 21, 351-356 (1978)

2. W. Cuypers, N. Van Gestelb, A. Voeta, J.P. Kruthb, J. Mingneaua, P. Bleysc, Optical measurement techniques for mobile and large-scale dimensional metrology, Opt. Laser Eng. 47, 292-300 (2009)

3. G.N. Peggs, P.G. Maropoulos, E.B. Hughes, A.B. Forbes, S. Robson, M. Ziebart, B. Muralikrishnan, Recent developments in large-scale dimensional metrology, in Proc. of the Institution of Mechanical Engineers, Part B: J. Eng. Manuf. 223, 571-595 (2009)

4. W.T. Estler, K.L. Edmundson, G.N. Peggs, D.H. Parker, Large-scale metrology - an update, CIRP Ann. Manuf. Technol. 51, 587-609 (2002)

5. F. Franceschini, M. Galetto, D. Maisano, L. Mastrogiacomo, Mobile spatial coordinate measuring system (MScMS) - Introduction to the system, Int. J. Prod. Res. 47, 3867-3889 (2009) 
6. T. Luhmann, S. Robson, S. Kyle, I. Harley, Close range photogrammetry (John Wiley \& Sons, New York, USA, 2006)

7. E.M. Mikhail, J.S. Bethel, J.C. McGlone, Introduction to modern photogrammetry (John Wiley \& Sons, New York, USA, 2001)

8. M. Galetto, L. Mastrogiacomo, B. Pralio, An innovative indoor coordinate measuring system for large-scale metrology based on a distributed IR sensor network, in Proc. of the ASME 2009 Int. Manufacturing Science and Engineering Conf. MSEC2009 West Lafayette, IN, USA (2009)

9. M. Galetto, B. Pralio, Optimal sensor positioning for large scale metrology applications, Precision Eng. 34, 563-577 (2010)
10. T. Svoboda, D. Martinec, T. Pajdla, A Convenient multicamera self-calibration for virtual environments. Presence: Teleoper. Virtual Environ. 14, 407-422 (2005)

11. ISO/IEC Guide 98-e:2008: Uncertainty of measurement Part 3: Guide to the expression of uncertainty in measurement (GUM:1995) (International Organization for Standardization, Geneva, Switzerland, 2008)

12. VDI/VDE 2634, Part 1, Optical 3D measuring systems and imaging systems with point-by-point probing (Beuth Verlag, Berlin, 2002)

13. VIM. International vocabulary of basic and General terms in metrology (International Organization for Standardization, Geneva, Switzerland, 2004) 